Parasitology

cambridge.org/par

\section{Research Article}

Cite this article: Barratt JLN, Sapp SGH (2020). Machine learning-based analyses support the existence of species complexes for Strongyloides fuelleborni and Strongyloides stercoralis. Parasitology 147, 1184-1195. https://doi.org/10.1017/S0031182020000979

Received: 18 March 2020

Revised: 12 May 2020

Accepted: 6 June 2020

First published online: 16 June 2020

\section{Key words:}

Genetics; genotypes; machine learning population structure; species complex; Strongyloides fuelleborni; Strongyloides stercoralis

\section{Author for correspondence:}

Joel L. N. Barratt, E-mail: jbarratt@cdc.gov; joelbarratt43@gmail.com and Sarah G. H. Sapp, E-mail: xyz6@cdc.gov

\title{
Machine learning-based analyses support the existence of species complexes for Strongyloides fuelleborni and Strongyloides stercoralis
}

\section{Joel L. N. Barratt ${ }^{1,2}$ (i) and Sarah G. H. Sapp ${ }^{1}$ (i)}

${ }^{1}$ Division of Parasitic Diseases and Malaria, Centers for Disease Control and Prevention, Parasitic Diseases Branch, Atlanta, USA and ${ }^{2}$ Oak Ridge Associated Universities, Oak Ridge, Tennessee, USA

\begin{abstract}
Human strongyloidiasis is a serious disease mostly attributable to Strongyloides stercoralis and to a lesser extent Strongyloides fuelleborni, a parasite mainly of non-human primates. The role of animals as reservoirs of human-infecting Strongyloides is ill-defined, and whether dogs are a source of human infection is debated. Published multi-locus sequence typing (MLST) studies attempt to elucidate relationships between Strongyloides genotypes, hosts, and distributions, but typically examine relatively few worms, making it difficult to identify population-level trends. Combining MLST data from multiple studies is often impractical because they examine different combinations of loci, eliminating phylogeny as a means of examining these data collectively unless hundreds of specimens are excluded. A recently-described machine learning approach that facilitates clustering of MLST data may offer a solution, even for datasets that include specimens sequenced at different combinations of loci. By clustering various MLST datasets as one using this procedure, we sought to uncover associations among genotype, geography, and hosts that remained elusive when examining datasets individually. Multiple datasets comprising hundreds of S. stercoralis and S. fuelleborni individuals were combined and clustered. Our results suggest that the commonly proposed 'two lineage' population structure of $S$. stercoralis (where lineage A infects humans and dogs, lineage B only dogs) is an over-simplification. Instead, S. stercoralis seemingly represents a species complex, including two distinct populations over-represented in dogs, and other populations vastly more common in humans. A distinction between African and Asian S. fuelleborni is also supported here, emphasizing the need for further resolving these taxonomic relationships through modern investigations.
\end{abstract}

\section{Introduction}

Strongyloidiasis affects approximately 370 million people in more than 70 countries, mostly in tropical and sub-tropical regions (Olsen et al., 2009; Bisoffi et al., 2013). Strongyloides infections in humans are typically caused by two species; predominantly Strongyloides stercoralis Stiles and Hassall 1902, with a smaller proportion caused by Strongyloides fuelleborni von Linstow, 1905. Most Strongyloides species exhibit high host specificity (Speare, 1989) but $S$. stercoralis infections have been reported in humans, dogs, cats and non-human primates. In particular, the role of dogs as reservoirs of zoonotic $S$. stercoralis has been a matter of contention historically and remains unclear today. Originally thought to infect humans exclusively, a nematode indistinguishable from S. stercoralis was identified in a dog in China in the early 1900s (Fuelleborn, 1914), raising questions as to whether S. stercoralis was responsible for both human and canine strongyloidiasis. The taxonomic separation of Strongyloides infecting humans and dogs was suggested on the basis of experimental and epidemiological evidence (Brumpt, 1922; Augustine, 1940; Jaleta et al., 2017), but this distinction has not been adopted. Even today, the epidemiology of canine strongyloidiasis remains poorly understood, and strongyloidiasis is generally considered a rare disease of dogs outside of East and Southeast Asia and the USA (Kreis, 1932; Galliard, 1951a). Reports of S. stercoralis or S. stercoralis-like species in domestic cats further complicates the question surrounding possible zoonotic reservoirs and Strongyloides species diversity (Chandler, 1925; Thamsborg et al., 2017; Wulcan et al., 2019).

Strongyloides stercoralis is currently thought to possess a population structure consisting of lineages A and B (Jaleta et al., 2017; Nagayasu et al., 2017; Barratt et al., 2019b). Humans and dogs are considered permissive hosts of $S$. stercoralis lineage A which is genetically characterized by possession of hyper-variable region (HVR)-IV haplotype A in most isolates, with the exception of some recently described Chinese S. stercoralis (Zhou et al., 2019). On the other hand, lineage B seemingly infects only dogs and invariably possesses HVR-IV haplotype $B$. However, fragmentary experimental evidence from early investigators suggests a more complicated population structure than the recently proposed 'two lineage' model implies (Nagayasu et al., 2017).

The less-common zoonotic species, S. fuelleborni (subsp. fuelleborni), is a non-human primate specialist (Pampiglione and Ricciardi, 1971, 1972; Hira and Patel, 1980; Nutman, 2017; 
Thanchomnang et al., 2017). Its global population structure has not been extensively studied and it is not known whether the ability to infect humans varies among populations. This variation might contribute to the relative geographic restriction of $S$. fuelleborni (subsp. fuelleborni) infections in humans, which are nearly exclusive to sub-Saharan Africa despite the broader occurrence of S. fuelleborni (subsp. fuelleborni) in primates in other parts of the Old World. The enigmatic S. fuelleborni subsp. kellyi Viney, Ashford, and Barnish 1991 has only been reported from humans in Papua New Guinea, and its relation to primate S. fuelleborni is ambiguous, possibly representing another species altogether (Dorris et al., 2002).

The broad geographic range, complicated taxonomic history, and possible differences in host permissibility raise questions about whether $S$. fuelleborni represents a complex of species with varying degrees of transmissibility to humans (Hung and Höppli, 1923; Sandground, 1925; Augustine, 1940; Premvati, 1959; Little, 1966a). Current taxonomic nomenclature does not distinguish between S. fuelleborni from Asian and African primates (Ashford and Barnish, 1989; Hasegawa et al., 2010). Like for S. stercoralis, modern genetic approaches could greatly aid in reevaluating the diversity, host and geographic associations of this species.

Hasegawa et al. (2009) proposed HVRs I to IV of the $18 \mathrm{~S}$ rDNA as useful markers for Strongyloides species diagnosis, especially HVR-IV, based on the observation that its nucleotide arrangements are mostly species specific'. HVR-I and HVR-IV are now routinely used in Strongyloides genotyping surveys (Barratt et al., 2019b; Zhou et al., 2019). The mitochondrial cytochrome c oxidase subunit 1 (cox1) locus of Strongyloides spp. is hypervariable and has been used to investigate genetic variation within and among Strongyloides populations by phylogenetic analysis and/or sequence clustering (Jaleta et al., 2017; Frias et al., 2018; Barratt et al., 2019b). When used in various combinations, these three multi-locus sequence typing (MLST) markers (cox1, HVR-I and HVR-IV) have demonstrable genotyping utility. However, Strongyloides genotyping surveys undertaken to date have used various combinations of these three loci, with some examining only one (Frias et al., 2018), or two loci (Sato et al., 2007; Hasegawa et al., 2009; Thanchomnang et al., 2017). In two recent surveys all three loci were examined (Jaleta et al., 2017; Zhou et al., 2019), and subsequently these three loci were used in a next-generation sequencing approach to attempt to identify all Strongyloides genotypes present in a single sample, directly from faecal DNA extracts (Barratt et al., 2019b; Beknazarova et al., 2019). These studies, which aimed to elucidate relationships between Strongyloides genotypes, hosts and geographic distributions, have led to an abundance of MLST data in public databases, representing hundreds of individual worms.

Individual published MLST studies usually analysed relatively small numbers of worms (often less than 100) from small numbers of hosts and locations, making it difficult to identify clear patterns within these populations. In addition, integrating MLST data from these various studies into a single large dataset is also impractical because the studies sometimes examine overlapping, but different combinations of loci. This means that phylogenetic and other analytic approaches cannot be utilized effectively to explore such datasets in an integrative manner.

A recently described unsupervised machine learning (ML) procedure that calculates a distance statistic from MLST data for downstream clustering, even for datasets composed of different loci and combinations thereof, produced at different timepoints, may offer a solution to this problem. This approach constitutes a novel population genetics tool comprising an ensemble of two ML algorithms (Barratt et al., 2019a). As input, this method requires a set of user-defined haplotypes from large population datasets. An advantage of this method over traditional sequence analysis approaches (e.g. phylogeny) is its ability to calculate distances from MLST data, even when the genotype of some specimens in the dataset is not completely defined (e.g. in the absence of data for a particular marker). Such an approach could facilitate the integration of MLST data for Strongyloides from multiple studies into a single analysis, even though the marker combinations vary among studies. Additionally, this method has an advantage that it can address the challenge of heterozygosity that might be encountered at nuclear loci in sexually reproducing eukaryotes (Barratt et al., 2019a). Thus, data for heterozygous individuals could also be retained for analysis.

In the present study, we applied this ML method to all publicly available MLST datasets for S. stercoralis and S. fuelleborni. We integrated these data to increase our ability to detect novel population-level associations. We hypothesized that the use of this method would enable the identification of relationships among Strongyloides genotypes, hosts and geographic distributions, that were not evident using smaller datasets individually. We propose that this approach could aid in resolving taxonomic questions and controversies surrounding Strongyloides species.

\section{Materials and methods}

\section{Data selection}

All available sequences of $S$. stercoralis and S. fuelleborni $18 S$ HVR-I, 18S HVR-IV $(n=218)$ and mitochondrial cox1 $(n=789)$ were obtained from GenBank (accessed August of 2019). Strongyloides sp. cox1 sequences obtained from Bornean slow lorises (Nycticebus borneanus, $n=18$ ) were also included for analysis, as they share a relatively close phylogenetic relationship with $S$. stercoralis and S. fuelleborni despite representing a distinct group (Frias et al., 2018). In cases where the HVR-I, HVR-IV and/or cox1 sequences were available from the same individual worm, the complete or partial genotype of these worms was recorded (Supplementary File S1, Tabs B and E, column F) after the genotyping approach by Barratt et al. (2019b) and Jaleta et al. (2017). In instances where specimens were genotyped using polymerase chain reaction (PCR) products amplified directly from stool (Barratt et al., 2019b; Beknazarova et al., 2019), only specimens obtaining a single haplotype for each locus were included in this analysis. The rationale for this criterion was that for amplicons sequenced directly from stool where multiple haplotypes were detected, the underlying genotype of individual worms cannot be elucidated. Sequences in GenBank possessing International Union of Pure and Applied Chemistry (IUPAC) ambiguity codes were unphased into their underlying haplotypes only if a sequence supporting the existence of both possible haplotypes was available in GenBank. If only one of two possible haplotypes were supported by other haplotypes in GenBank for such a sequence, the IUPAC code was changed in favour of the supported haplotype. If there was no support for either of the underlying haplotypes for a sequence in GenBank possessing IUPAC codes, this part of the sequence was trimmed off from the ambiguous base onwards, in the direction that maximized the final length of the sequence. This analysis was inclusive of all S. stercoralis and S. fuelleborni sequences available in GenBank that met these criteria, irrespective of their host origin, and included specimens from humans, non-human primates, dogs and cats (Supplementary File S1, Tabs B and E, column D). Due to the limited sequence data available for S. fuelleborni kellyi, it was excluded from this analysis.

Genotypes were also constructed for five S. fuelleborni specimens from Japanese macaques, using all three MLST markers (i.e. HVR-I, HVR-IV and cox1). These genotypes were constructed from five cox 1 sequences generated in one study 
(Hasegawa et al., 2010), and 18S rDNA sequences published in different studies presumably from different worms (Sato et al., 2007; Hasegawa et al., 2009). Therefore, 'synthetic' genotypes were constructed from these sequences to represent $S$. fuelleborni from the five Japanese macaques (Macaca fuscata). The synthetic genotypes were constructed in this way based on the observation that each of these $18 \mathrm{~S}$ rDNA sequences was generated from worms collected from different Japanese macaque populations, yet all sequences are identical (GB: AB272235.1, AB453317.1, AB453318.1, AB453319.1). This supports that they represent the haplotypes found in all Japanese macaques (Sato et al., 2007; Hasegawa et al., 2009). However, because these genotypes were based on human inference, classification was performed twice on the S. fuelleborni dataset; once including and once excluding these five 'synthetic' Japanese S. fuelleborni specimens.

\section{Extracting cox1 and 18s haplotypes from published Strongyloides genomes}

Genome sequences of S. stercoralis (Kikuchi et al., 2016) (36 genomes in total) were downloaded from the GenBank SRA database (Supplementary File S1, Tab B, column E) and the raw Illumina reads were subjected to a workflow designed in Geneious Prime (version 11: www.geneious.com). This workflow performed removal of Illumina adapter sequences (using BBDuk - default parameters) and filtering for quality (minimum PHRED score: 20 and minimum read length: 50 bases). Trimmed reads were mapped to references of HVR-I (GenBank: AF279916.2), HVR-IV (GenBank: AF279916.2), and cox1 (GenBank: MK463927.1) using Geneious mapper (default parameters) and the consensus of each mapped assembly was extracted for inclusion in this analysis.

\section{Haplotype definitions}

Haplotypes were identified in each specimen using a recently described genotyping system (Jaleta et al., 2017; Barratt et al., $2019 b$ ) with some modifications (Fig. 1). Briefly, for the purposes of this study, sequences of the $\sim 434 \mathrm{bp}$ fragment of HVR-I were divided into four segments, sequences of the $\sim 260 \mathrm{bp}$ fragment of HVR-IV were divided into three segments, and a 217 bp region of cox1 defined elsewhere (Beknazarova et al., 2019; Barratt et al., $2019 b$ ) was divided into nine segments (A1 to A3, B1 to B3 and $\mathrm{C} 1$ to $\mathrm{C} 3$ ), so that each of these sub-segments was considered a distinct locus. The rationale for dividing the sequences into these segments is based on extensive testing of the ML approach for performance optimization. These tests indicated that performance is best when the number of haplotypes at a given locus is between 10 and 20, and preferably less than 30 (https://github. com/Joel-Barratt/Eukaryotyping). The reasons for this are explained in detail in Supplementary File S2, pages 4-7. The sequence of each haplotype after the division of the three MLST markers into segments (resulting in a final panel of 16 markers) is provided in Supplementary File S2, Appendix (part A).

\section{Assessment of population structure}

Genotypes were assigned to each specimen using a Geneious workflow developed by the study team. Firstly, the Strongyloides sequences from individual worms were merged into a sequence list and then compared to a BLASTN database containing the FASTA sequences provided in Supplementary File S2, Appendix part A. The results were exported from Geneious in text format (one file for each specimen) and this result was converted to the format shown in Supplementary file S1 (Tabs A and D). The ML procedure (Barratt et al., 2019a) was applied to the resultant Strongyloides dataset using the scripts and instructions available here: https://github.com/Joel-Barratt/Eukaryotyping. Briefly, the haplotype data sheets provided in Supplementary file S1 (Tabs A and D) were exported as .txt files; these files were used directly as the input for the $\mathrm{R}$ scripts. This input included 138 specimens from S. fuelleborni and Strongyloides sp. 'loris', and 764 from S. stercoralis.

The ML procedure performs an unsupervised similarity-based classification task; it assesses whether any two specimens are related or unrelated on the basis of their genotype. The algorithms underpinning this method do not require that the genotype of every specimen be defined in the same manner; specimens are not required to be sequenced at the same markers (Barratt et al., 2019a), although, realistic minimum data requirements must be set by the user prior to analysis (https:/github.com/ Joel-Barratt/Eukaryotyping). For example, it would be unrealistic to expect that a specimen would be accurately classified in the event that only 20 bases of a single locus were available for this specimen. In this study, specimens were only retained for classification if they met at least one of two minimum data availability criteria. Firstly (1), when cox 1 was the only sequence available for a specimen, or when data was not obtained for Part B of HVR-IV, the availability of nine out of the nine cox 1 segments was required for a specimen to be included in the analysis. Secondly (2), if the only cox 1 sequence available for a specimen was truncated (i.e. partially overlapping with the $217 \mathrm{bp}$ region of coxl being analysed here), the specimen could still be analysed provided its cox 1 sequence overlapped with this $217 \mathrm{bp}$ region by a minimum of seven out of nine segments. However, specimens with a truncated cox 1 sequence were only retained in this analysis if part B of HVR-IV was also available for that specimen. This requirement considers the pertinent observations of Hasegawa et al. (2009), regarding HVR-IV, indicating that its nucleotide arrangements are mostly species specific'. It also considers that Part B of HVR-I can differentiate lineages A and B of S. stercoralis (Nagayasu et al., 2017; Barratt et al., 2019b). Therefore, part B of HVR-IV was set as a supplementary data requirement to compensate for information lost in the event of a truncated cox 1 sequence. Data for HVR-I and/or additional parts of HVR-IV were considered in the analysis if available for a specimen, but data for these loci were not an absolute requirement for retaining specimens in this analysis.

The classification was performed for S. stercoralis and S. fuelleborni separately using an epsilon value of 0.05 for Plucinski's naïve Bayes classifier (refer to: https://github.com/Joel-Barratt/ Eukaryotyping), and the resultant pairwise distance matrices (a pairwise matrix is the standard output of our ML method Supplementary File S1, Tabs C and F) were clustered. Clustering was performed using the agglomerative nested approach in the 'agnes' $\mathrm{R}$ package, utilizing Ward's clustering method as described here (Barratt et al., 2019b). The 'ggtree' R package was then used to generate cluster dendrograms. Images of relevant hosts were obtained from PhyloPic (http://phylopic. org) or prepared in-house at the Centers for Disease Control and Prevention (CDC) for annotation of dendrograms. Images were rendered using the freely available GNU Image manipulation program (https://www.gimp.org).

\section{Rationale for study design}

Strongyloides coxl sequences are considered hypervariable; this variability relates to single nucleotide polymorphisms (SNPs). As cox 1 is also encoded on the mitochondrion, it is not subject to heterozygosity. These features make coxl conducive to an analysis by phylogenetic methods or clustering based on sequence similarity (Jaleta et al., 2017; Barratt et al., 2019b; Beknazarova 


\section{Strongyloides spp. SSU rDNA HVR-I haplotypes}

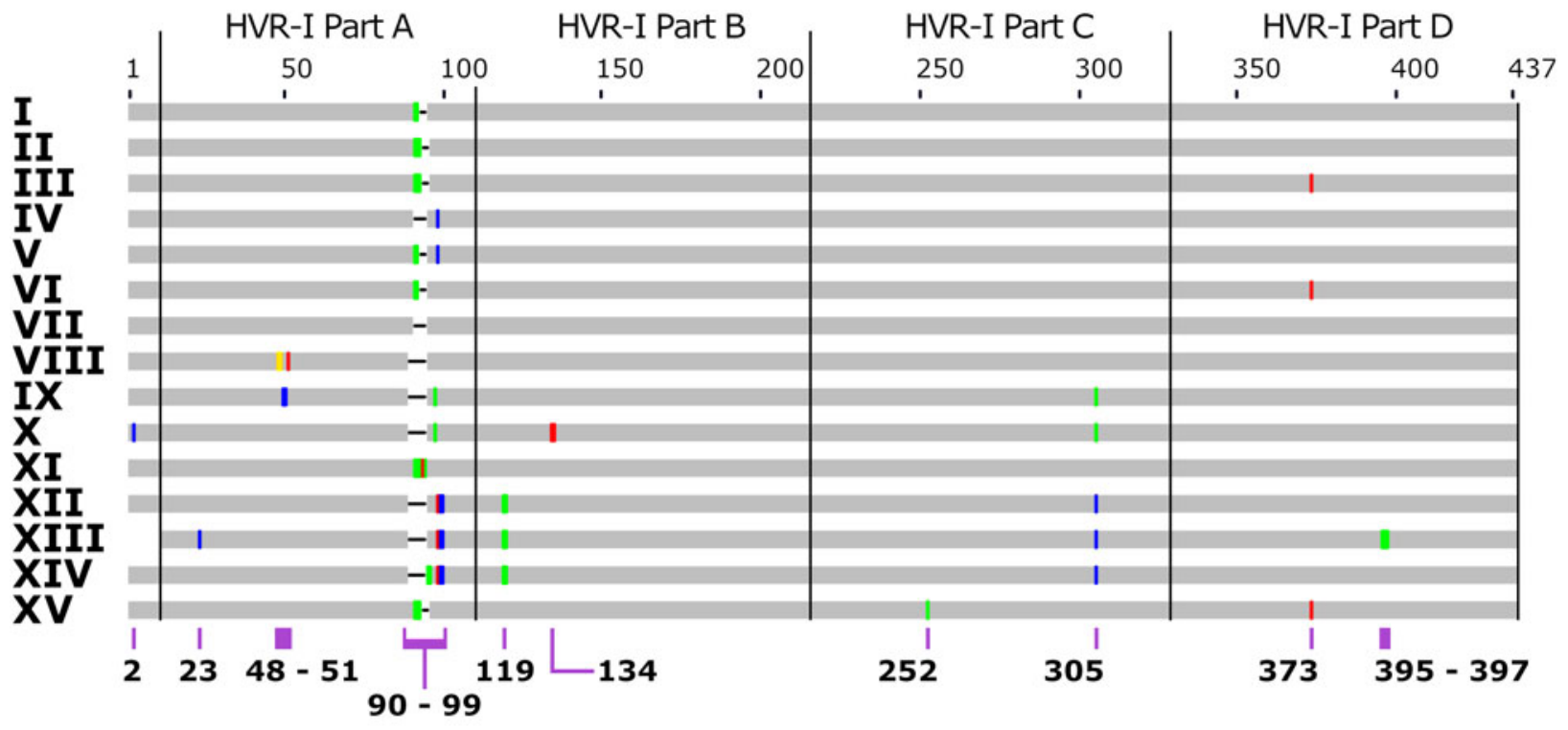

Strongyloides spp. SSU rDNA HVR-IV haplotypes

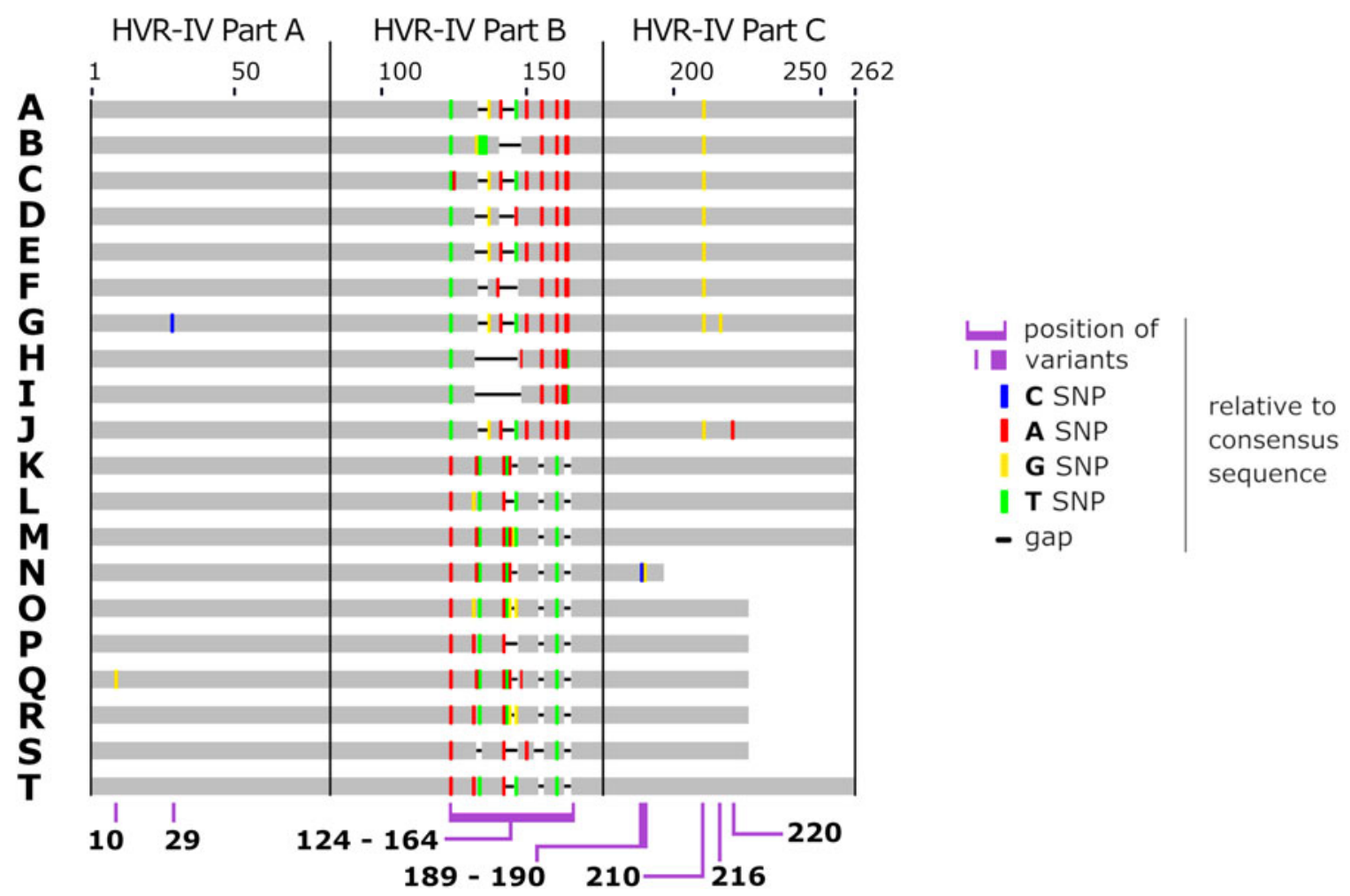

Fig. 1. Schematic of the Strongyloides genotyping scheme referenced here. Graphical representation a Strongyloides sp. genotyping scheme after the description of Barratt et al. (2019b) and Jaleta et al. (2017). This scheme was expanded here to include haplotype XV of 18S HVR-I (GenBank Accession: MT436714), identified in genomes sequenced from Japanese humans by Kikuchi et al. (2016). For the purposes of this study, note that the haplotypes originally defined after the description of Barratt and colleagues were split into smaller segments as shown here.

et al., 2019; Zhou et al., 2019). Alternatively, the $18 \mathrm{~S}$ rDNA locus may be heterozygous in some individuals of $S$. stercoralis (see Zhou et al., 2019), which is an important confounding factor for phylogenetic methods. Within the Strongyloides genus, the $18 \mathrm{~S}$ rDNA locus is less diverse than cox 1 but it contains nucleotide insertions and deletions (indels) that represent important variants for differentiating Strongyloides species and genotypes. Most phylogenetic algorithms typically treat indels as missing data (Truszkowski and Goldman, 2016; Donath and Stadler,
2018), and variability in indel handling between various multiple sequence aligners prior to phylogeny can produce markedly different results (Golubchik et al., 2007; Ashkenazy et al., 2014). For this reason, algorithms such as Gblocks are often used to identify and remove regions of the alignment containing gaps, making this data more amenable to phylogenetic applications (Castresana, 2000). Unfortunately, this practice would result in a loss of meaningful information when analyzing Strongyloides $18 \mathrm{~S}$ rDNA HVRs. Furthermore, Strongyloides genotyping surveys 
published in the last decade display only some consistency in the combinations of the three widely used genotyping loci examined (Hasegawa et al., 2009, 2010; Schär et al., 2014; Laymanivong et al., 2016; Jaleta et al., 2017; Thanchomnang et al., 2017, 2019; Frias et al., 2018; Barratt et al., 2019b; Beknazarova et al., 2019). This makes comparative analyses of distinct datasets difficult when using phylogenetic and/or sequence clustering methods. These limitations represent the main impetus for the design of this study, specifically; the ML procedure that was designed to overcome these challenges (Barratt et al., 2019a) (Supplementary File S2).

\section{Results}

\section{Data filtering and detection of novel 18s rDNA haplotypes}

After filtering the combined MLST dataset according to the minimum data availability requirements, data from 704 of the original 764 genotyped S. stercoralis specimens were retained. For S. fuelleborni, data from 133 of the 138 genotyped specimens were retained including all sequences from Strongyloides sp. 'Loris'. These specimens had varying combinations of HVR-I, HVR-IV and cox1 data available; the markers available for each individual specimen that was retained for analysis are provided in Supplementary File S1, Tabs B and E, column F.

A novel S. stercoralis $18 \mathrm{~S}$ rDNA HVR-I haplotype was identified in previously published genome datasets (Kikuchi et al., 2016). This $18 \mathrm{~S}$ rDNA haplotype was detected in specimens from Okinawa, Japan and was assigned to haplotype XV, which contains a unique SNP at position 252 relative to the alignment shown in Fig. 1. The sequence of haplotype XV is identical to that of HVR-I haplotype III at all other base positions. The Strongyloides sp. genotyping scheme previously described (Jaleta et al., 2017; Barratt et al., 2019b; Beknazarova et al., 2019), was thus expanded to include this novel type (GenBank Accession: MT436714). The typing scheme used here (Barratt et al., 2019b; Beknazarova et al., 2019), differs from that described by Zhou et al. (2019), who described HVR-IV haplotype C, which is identical to haplotype E reported by Beknazarova et al. (2019) (Fig. 1).

\section{The population structure of Strongyloides stercoralis}

The final filtered $S$. stercoralis dataset includes sequences from specimens representing all inhabited continents. However, S. stercoralis sequences from specimens collected in Japan and some Southeast Asian countries were more highly represented (Supplementary File S2, page 13). MLST data from S. stercoralis collected from humans were more highly represented than data collected from dogs (Table 1, Fig. 2). Following classification using ML, the dataset was divided into 7 distinct genetic clusters, each representing a proposed sub-population of $S$. stercoralis (Fig. 2). The vast majority of $S$. stercoralis from dogs were assigned to one of two populations based on the MLST data available, represented by clusters 4 and 5 (129 of 146 S. stercoralis from dogs were assigned to these clusters; $88.4 \%)$. Most S. stercoralis specimens of human origin $(550 / 554,99.3 \%)$ were assigned to clusters 1, 2, 3, 6 or 7 based on their genotype (Table 1). All specimens assigned to cluster 5 (92/92) were derived from dogs as were $86 \%$ of specimens (37/43) assigned to cluster 4 . Two specimens assigned to cluster 4 were obtained from cats and only 4 specimens assigned to this cluster were from humans $(90.7 \%$ of cluster 4 included S. stercoralis collected from dogs or cats). Overall, $95.5 \%$ of $S$. stercoralis assigned to clusters 4 and 5 based on their MLST genotype had been isolated from dogs. Given the geographical sampling bias towards specimens collected in Southeast Asia and Japan (Supplementary File S2, pages 13 and 14), no
Table 1. Frequency of $S$. stercoralis from dogs and humans assigned to each genetic cluster and their respective $\chi 2 P$ values

\begin{tabular}{lllll}
\hline & Humans & Dogs & P-value & $\begin{array}{c}\text { Over-represented } \\
\text { host in each } \\
\text { cluster }\end{array}$ \\
\hline Cluster_1 & 138 & 2 & 0.000 & Humans \\
\hline Cluster_2 & 56 & 1 & 0.000 & Humans \\
\hline Cluster_3 & 236 & 3 & 0.000 & Humans \\
\hline Cluster_4 & 4 & 37 & 0.000 & Dogs \\
\hline Cluster_5 & 0 & 92 & 0.000 & Dogs \\
\hline Cluster_6 & 88 & 2 & 0.000 & Humans \\
\hline Cluster_7 & 32 & 9 & 0.000 & Humans \\
\hline TOTAL & 554 & 146 & & \\
\hline
\end{tabular}

Note: Strongyloides stercoralis from humans were rarely assigned to cluster 4 and never to cluster 5 . Strongyloides stercoralis from dogs, were vastly more common in clusters 4 and 5 ( $88.4 \%$ of cases), though $6.2 \%$ S. stercoralis from dogs were assigned to cluster 7 . Boxes containing numeric values are shaded according to their frequency, with the highest frequencies shown in black and the lowest frequency (zero) in white.

attempt was made to statistically validate possible associations between genetic cluster assignment and the geographic origin of $S$. stercoralis specimens. An exception to this is genetic cluster 5 , where 90 of 92 specimens assigned to this cluster (98\%) were collected from dogs in Cambodia or Myanmar.

\section{The population structure of Strongyloides fuelleborni}

The $S$. fuelleborni dataset retained for classification includes specimens collected mostly from parts of Southeast Asia and Africa, one specimen from India, and five specimens from Japan. Overall, the study population included 9 specimens from humans and 124 specimens from non-human primates. Long-tailed macaques (Macaca fascicularis) were the most highly sampled host among the specimens included in this analysis. The S. fuelleborni dataset also included 18 specimens obtained from Bornean slow lorises (Nycticebus borneanus). For precise numbers of specimens retained for analysis from each host and country refer to Supplementary File S2, Tables S4 to S7. The genotypes constructed for the five $S$. fuelleborni specimens from Japanese macaques possess all markers (i.e. HVR-I, HVR-IV and cox1) but these genotypes were generated from five coxl sequences from one study (Hasegawa et al., 2010), and 18S rDNA sequences obtained in different studies from different worms. Regarding these 'synthetic' genotypes from S. fuelleborni infecting Japanese macaques, classification was performed twice on the S. fuelleborni dataset; once including and once excluding these five 'synthetic' specimens. Irrespectively, the presence or absence of these specimens during classification had very little impact on the S. fuelleborni population structure (Supplementary File S2, Appendix part D).

Following classification, the S. fuelleborni specimens (plus Strongyloides sp. from slow lorises) were divided amongst 7 clusters, each representing a distinct population (Fig. 3). Clusters 1 and 2 as well as clusters 6 and 7, showed no clear association with a particular primate host species. Cluster 5 is exclusively occupied by the Strongyloides sp. from lorises, though the distinctness of this group was previously reported (Frias et al., 2018). Cluster 4 is occupied by only a small number of inferred genotypes from one host species (Japanese macaques), and the specimens obtained from long-tailed macaques assigned to cluster 3 come from a single study surveying only this primate species in Thailand and Laos (Thanchomnang et al., 2017). Therefore, the possibility that this is a geographic association as opposed to a host association cannot be excluded. Generally, the data support 


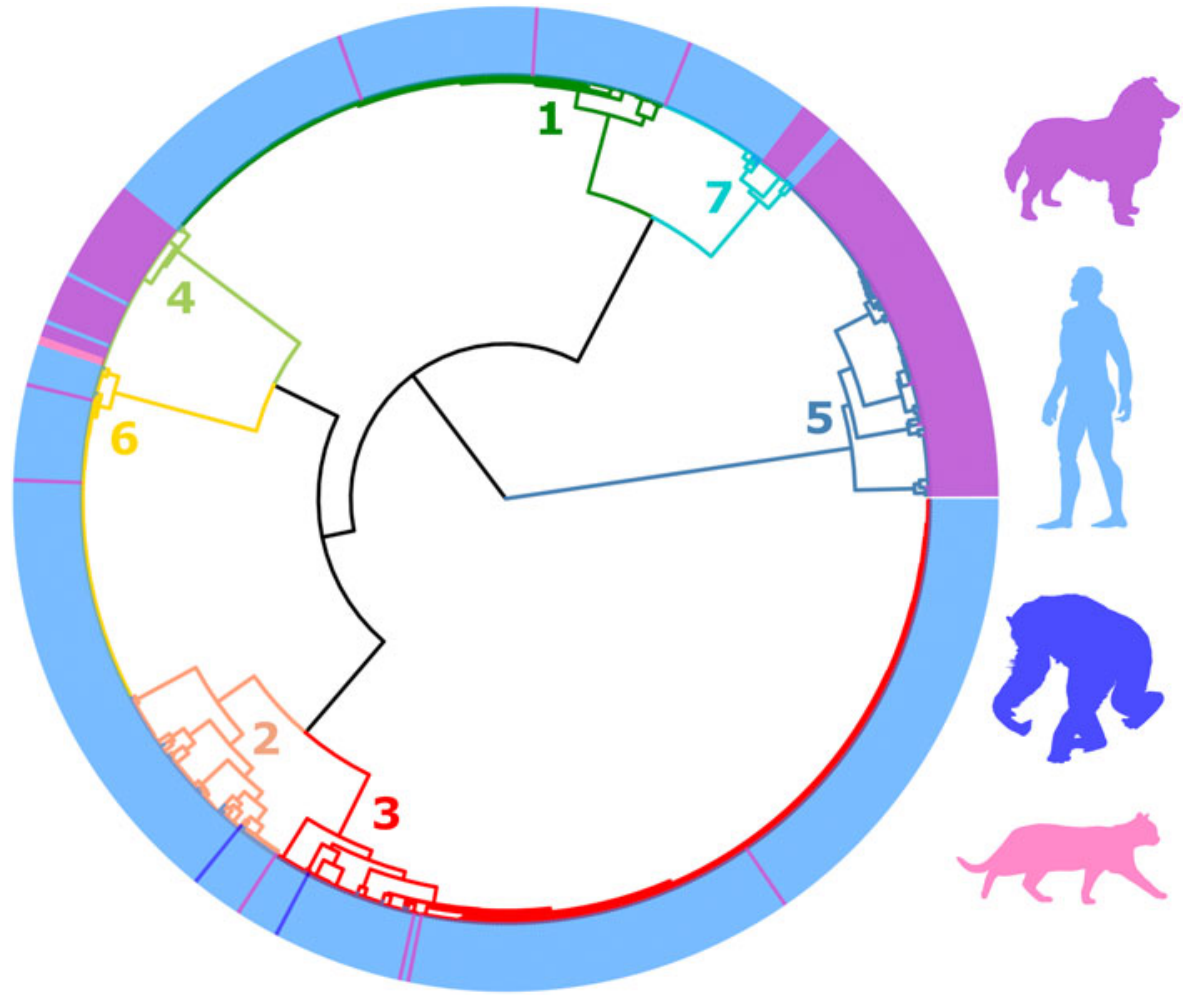

Fig. 2. Dendrogram of clustered distances generated from genotyped Strongyloides stercoralis using ML. This dendrogram was divided into seven distinct clusters. Branches are coloured according to their cluster number and are simply used to differentiate branches. Coloured peripheral bars indicate the host species from which the genotyped S. stercoralis were collected from; either a dog, human, chimpanzee or cat. The colour of these respective silhouettes matches the peripheral bars. For the identity of each specimen in this dendrogram refer to Supplementary File S2, Appendix part B. Cluster 5 is exclusively occupied by $S$. stercoralis infecting dogs. Specimens assigned to Cluster 4 were more frequently collected from dogs, though were also found in humans and cats. S. stercoralis from chimpanzees were assigned to clusters 2 and 3 , though these were less frequently observed in these samples. S. stercoralis from clusters $1,2,3,6$ and 7 were more frequently collected from human samples than other hosts. The geographic origin of sequenced specimens included in this dendrogram is shown in Supplementary File S1, Tab B. a population structure for S. fuelleborni based on associations among genotype and geography; clusters 1 and 2 including $S$. fuelleborni from Africa, clusters 3 and 4 including S. fuelleborni from Southeast Asia and Japan (respectively), and clusters 6 and 7 including S. fuelleborni from Malaysian Borneo, which is suggestive of allopatric speciation.

\section{Discussion}

The ability to integrate large sequence datasets from multiple studies composed of specimens with varying combinations of MLST markers amplified, and specimens that are heterozygous, represents an important advantage of the ML procedure employed here over traditional methods utilized in the field of population genetics. Furthermore, the way in which haplotypes are defined using our ML procedure avoids problems associated with the treatment of indels that are inherent in many phylogenetic methods; indels (alignment gaps) are often treated as missing data in phylogenetic analyses and are excluded altogether (Truszkowski and Goldman, 2016; Donath and Stadler, 2018). This exclusion of alignment gaps is problematic for the analysis of Strongyloides HVRs because these loci contain informative indels that differentiate haplotypes. Indels do not represent a problem for the ML approach used here which represents another advantage of this approach over phylogenetic methods. This method facilitated the inclusion of hundreds of genotyped S. stercoralis specimens from multiple studies in a single analysis, despite that many published genotyping studies examined different, yet overlapping, combinations of markers. Therefore, this analysis provides a broader view of potential host-associations and geographic patterns that exist in populations of these worms than could be provided in previous studies given the data analysis challenges mentioned above. The large number of specimens retained for analysis here meant that any observable trends are supported with greater statistical power than could be achieved within the smaller genotyping studies. Consequently, this ML procedure represents a powerful alternative to traditional phylogenetic methods. Using this approach, we provide evidence supporting that $S$. stercoralis represents a species complex and that African and s.E. Asian S. fuelleborni are distinct. These results are supported by a synthesis of scientific literature, focusing on observed patterns relating to host preference and geography reported on the basis of biological and experimental evidence published by pioneering investigators who were predominantly active in the early to middle 20th century. This synthesis highlights the agreement between our current analysis and the observations of these classic parasitologists who lacked the molecular tools required to help resolve these important taxonomic questions.

Since Fuelleborn's initial discovery of a Strongyloides infection in a Chinese dog, the status of dog-derived isolates as a valid species or as a variant of human S. stercoralis, and thus its zoonotic potential, has remained a subject of debate (Fuelleborn, 1914). While many authors regarded canine and human isolates as more or less morphologically identical strains with differing host specificity within the same species S. stercoralis (Hung and Höppli, 1923; Kreis, 1932), others recognized the potential existence of 'geographic races' or even suggested the separation of canine Strongyloides on experimental and epidemiological grounds in spite of negligible morphological differences (Brumpt, 1922; Augustine and Davey, 1939). Brumpt (1922) argued for the establishment of a new species Strongyloides canis on the basis of geographic disparities in prevalence, barriers in experimental cross-transmission, and reported developmental differences in vitro. As most early 20th century authors were averse to applying for specific status without important morphological differences (Chandler, 1925; Sandground, 1925; Goodey, 1926; Kreis, 1932), S. canis was never recognized as a valid name. However, the modern availability of genetic data - including our results - and the resulting shift in taxonomic dogmas allows this question to be revisited.

The body of experimental evidence demonstrates varying abilities of human-derived $S$. stercoralis from different geographic regions to establish infections in dogs. This could correspond with the 'spectrum' between human- and apparently 
Fig. 3. Dendrogram of clustered distances generated from genotyped specimens of Strongyloides fuelleborni and Strongyloides sp. from lorises using ML. This dendrogram was divided into seven distinct clusters. Branches are coloured according to their cluster number and the coloured peripheral bars indicate the host species from which the $S$. stercoralis specimens/ sequences were derived, either a human $(\mathrm{Hu})$, gorilla $(\mathrm{Go})$, chimpanzee $(\mathrm{Ch})$, baboon $(\mathrm{Ba})$, orangutan (Or), Long-tailed macaque (Lt), proboscis monkey (Pr), Silvered leaf monkey (SI), Slow loris (Lo), or Japanese macaque $(\mathrm{Jm})$. Black branches visualize the relationship between clusters. For the identity of each specimen in this dendrogram refer to Supplementary File S1, Appendix part C. Clusters 1 \& 2 include specimens from Africa. Cluster 3 includes specimens from Thailand and Laos, and a single specimen from an Indian human. Cluster 4 includes specimens collected in Japan. Clusters 5, 6 and 7 include specimens from Malaysian Borneo. This dendrogram supports that clusters 1 and 2 include $S$. fuelleborni genotypes that infect humans more commonly than the Strongyloides in other clusters.

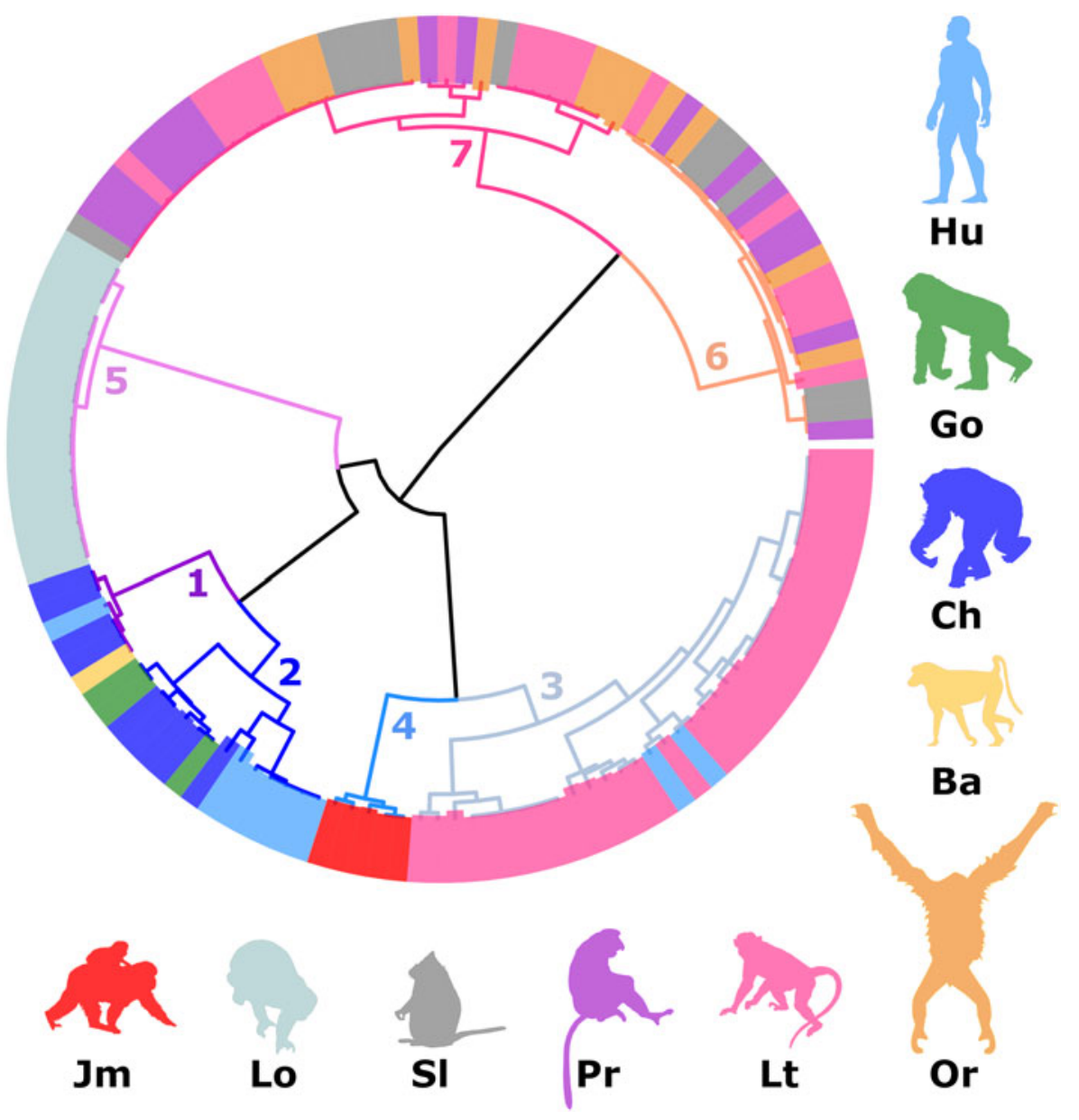

canine-restricted genetic lineages presented here (Fig. 2). In Fuelleborn's investigations (Fuelleborn, 1914, 1927), dogs developed only short duration infections (2-3 weeks) with humanderived Chinese $S$. stercoralis and were refractory to infection with East African S. stercoralis. Sandground successfully infected dogs with a USA human-derived strain, though the majority of dogs eventually self-cured after about 3-10 weeks and were refractory towards repeated exposures (Sandground, 1928; Galliard, 1939, 1951b). Galliard reported ease in infecting dogs with human Strongyloides from Vietnam (Galliard, 1939), but that dogs were more or less refractory to isolates from the West Indies and North Africa (Galliard, 1951a). Also, in his experiments, dogs that were not successfully infected with the African strain developed severe infections after exposure to the Vietnamese strain.

Sandosham (1952) reported short-term, unstable infection in a dog repeatedly inoculated with many larvae derived from former prisoners of war in Thailand, concluding that dogs could not have been the reservoir host for $S$. stercoralis in that setting despite their supposed abundant presence in the prison camps (Sandosham, 1952). Many years later, attempts to infect laboratory dogs with S. stercoralis derived from a human patient infected in Southeast Asia yielded mixed results; in one dog, a chronic infection lasting at least 15 months was established, but in four others, fecal larval counts peaked at 3 weeks and dropped off drastically after that (Grove and Northern, 1982). A similar pattern of unstable, transient patent infections has been noted previously in human volunteers infected with other animal Strongyloides species, such as Strongyloides procyonis (raccoons) and Strongyloides ransomi (swine), further suggesting that host adaptation strongly influences the duration of infection and larval output (Freedman, 1991). Reciprocally, Augustine and Davey (1939) failed to infect a human volunteer, guinea pigs and cats with filariform larvae reared from a naturally-infected dog in Massachusetts but readily infected both young and aged dogs, which developed long-lasting patent infections.

Clearly, historical workers had differing levels of success infecting dogs, and one varying factor was the geographic origin of isolates. Though fragmentary, it is interesting to compare the geographic origins of parasites used in dog infection trials to the emerging picture of the $S$. stercoralis global population structure shown here. For example, dogs were found to be refractory or developing very short-lived infections when exposed to humanderived isolates of $S$. stercoralis from China, East Africa, North Africa and the West Indies (Fuelleborn, 1914; Galliard, 1950; Galliard and Berdonneau, 1953). Notably, the lineages of S. stercoralis, represented by clusters $1,2,3$ and 6 were almost all obtained from human samples and known to occur in these locations (Fig. 2). Intermediate results (e.g. short-lived infections, low larval outputs, and/or resistance to reinfection in most dogs, with occasional chronic infections) were seen with North American and some Southeast Asian S. stercoralis strains (Sandground, 1925; Faust, 1933; Grove and Northern, 1982; Genta, 1989); these locations are represented by lineages of $S$. stercoralis assigned to genetic clusters 4 and 7 , where we observe genotypes that are seemingly capable of infecting both hosts, yet with the majority of specimens assigned to these clusters collected from dogs and humans, respectively (Fig. 2, Table 1). The sole trial where dogs were readily infected with no immunity to reinfection used a human-derived S. stercoralis from Vietnam (Galliard, 1939), which is in close geographic proximity to the origin of roughly half of all specimens assigned to cluster $7 \quad(n=20$, Myanmar and Thailand). In summary, the observed historical variations show some overlap with both the locations and host frequencies in our observed S. stercoralis clusters. 
Our analysis suggests a disparity between where human and canine S. stercoralis infections are occurring; infections caused by $S$. stercoralis assigned to cluster 5 are almost exclusive to Southeast Asia (98\%). Our analysis also indicates that different genotypes of $S$. stercoralis are more frequently reported in specific hosts (dogs are over-represented hosts in clusters 4 and 5), based on a $\chi^{2}$ analysis (Table 1 ). These observations were also extensively discussed by historical authors who commented on the marked disparity in the occurrence of canine and human S. stercoralis infections i.e. in places where $\operatorname{dog} S$. stercoralis infections occur commonly, human infections are rare, and vice versa. Outside of East and Southeast Asia, natural infections in dogs appeared to only occur in North America, and reports of dog infections were exceedingly rare to absent in areas where prevalence was high in humans (Brumpt, 1922; Faust, 1933; Augustine, 1940; Galliard, 1950; Sandosham, 1952; Genta and Grove, 1989). For example, not a single infection was detected in 528 dog faecal specimens during a survey in a highly endemic region of Colombia, where at least $14 \%$ of humans were $S$. stercoralis-positive (Faust and Giraldo, 1960); even if some infections were missed due to the use of flotation techniques, infection would still be very rare in dogs $v s$ humans. Recent case reports and molecular evidence show that natural canine infections also occur in Europe and Australia (Paradies et al., 2017; Basso et al., 2019; Beknazarova et al., 2019; Barratt et al., 2019b). However, an important caveat is that results based solely on microscopy of feces must be interpreted with caution as freeliving nematode larvae, common contaminants of specimens collected from the ground can easily be confused with Strongyloides spp. (Speare, 1989). Direct epidemiologic evidence for crosstransmissibility is limited, in part due to the apparent variability in host adaptation and geographic occurrence of these strains. In one instance, a kennel worker in the USA with no foreign travel acquired Strongyloides from dogs under his care; this strain was successfully used to infect laboratory dogs (Georgi and Sprinkle, 1974). A small study in an area of Japan considered endemic found no association in S. stercoralis infection status among owners and dogs (Takano et al., 2009). Overall, there are still many knowledge gaps in our understanding of canine strongyloidiasis as a zoonosis and its epidemiology, and interpretation will require further characterization of strains across a diverse geographic range.

While establishing the specific identity of canine Strongyloides will require further investigation, based on historical reports and our novel analysis, it appears that different $S$. stercoralis lineages display differences in infection frequency for canine and human hosts - which may exclude or drastically limit cross-transmission potential in some cases (Table 1). Given the epidemiological implications of this genetic variability and the now large body of evidence across many independent studies, it now seems appropriate to treat $S$. stercoralis as a species complex. For example, 'S. stercoralis sensu lato' could be useful for referring to all isolates, and 'S. stercoralis sensu stricto' could be considered for the human-genotypes represented in 6 of the 7 clusters associated with HVR-IV haplotype A (Fig. 2, excluding cluster 5). This would also include specimens assigned to genetic cluster 4 which seems associated with the $18 \mathrm{~S}$ rDNA genotype $\mathrm{VI}+\mathrm{A}$, and likely represents a canine adapted lineage of S. stercoralis sensu stricto. Subspecific ranks (e.g. S. stercoralis canis) were suggested nearly a century ago by Chandler for $S$. stercoralis of different animal hosts (Chandler, 1925). This seems reasonable, given the exclusive finding in canine hosts (100\% of cluster 5$)$, the restricted geographic range of $S$. stercoralis assigned to cluster 5 (98\% were from Southeast Asia), and the fact this lineage (associated with HVR-IV haplotype B) naturally clusters as an outgroup to all other S. stercoralis (Fig. 2).
An important outstanding question is whether the canine infections in certain human host-dominated populations represent transient, spurious, or incidental infections, or if there exists a substructure that is not clearly apparent due to undersampling of dog-derived genotypes from specific locations. Based on our analysis, dog-derived genotypes assigned to clusters 1-3 and 6 likely represent transient infections with strains originating from humans; this is supported by the experiments of Fuelleborn, Sanground and Galliard who infected dogs with human-derived $S$. stercoralis which often resulted in transient infections (Fuelleborn, 1914, 1927; Sandground, 1928; Galliard, 1939, 1951a, 1951b). However, for cluster 7 it seems possible that some additional population substructure exists. Cluster 7 contains a small 'sub-cluster' composed of eight cox1 sequences derived exclusively from $S$. stercoralis from Japanese dogs. Sequencing of HVR-I and/or HVR-IV from these worms, and the sampling of worms from additional dogs in the same geographic area that these specimens were collected, would provide greater clarity as to what this smaller 'sub-cluster' of Cluster 7 might represent.

The identity of Strongyloides spp. infecting cats is a more complex question, and it appears multiple species are involved, including S. stercoralis. Chandler first reported S. stercoralis in cats in India (Chandler, 1925), and some attempts to infect cats with $S$. stercoralis from dogs and humans have been successful; though infections were usually short-lived, and it appears that cats are competent but abnormal hosts for S. stercoralis (Sandground, 1928; Desportes, 1944; Wulcan et al., 2019). Strongyloides felis has been described from India and Australia, and is somewhat similar morphologically to $S$. stercoralis although no molecular data are available (Speare and Tinsley, 1986). Strongyloides planiceps occasionally infects cats but is dissimilar in its life cycle and morphologic characteristics to S. stercoralis (Thamsborg et al., 2017). A fourth species, Strongyloides tumefaciens, is of interest as it has been reported as causing colonic nodules, an unusual clinical presentation for Strongyloides (Thamsborg et al., 2017). However, the original description was incomplete, and a recent study cast doubt on the validity of S. tumefaciens. Strongyloides sp. extracted from nodules of necropsied cats were found to have cox 1 sequences that matched $S$. stercoralis. These sequences were assigned to genetic cluster 4 in this study (Fig. 2), suggesting that $S$. tumefaciens infections could simply be an unusual pathological presentation of S. stercoralis (Wulcan et al., 2019). There is clearly a need for further investigation into Strongyloides spp. of domestic cats, especially in regard to their zoonotic potential. The analytic approach presented here, along with morphological and biological characterization, should prove beneficial in answering these questions.

The other topic of interest in our study was the identity and population structure of $S$. fuelleborni as it relates to human and other primate infections. The validity and number of species infecting primates have been long debated. Genetic analyses, including the approach used here, have proven valuable in reconciling some of these viewpoints, as morphological comparisons have confounded historical investigations of Strongyloides taxonomy. This challenge mainly owes to the variability of characters and their interpretation - importantly, how much relative weight was given to which characteristics for species designations. For example, both Chandler and Little felt that morphologic features of the parasitic female were most reliable (Chandler, 1925; Little, 1966a), whereas Looss and Goodey placed more importance on the free-living adult stages (Looss, 1911; Goodey, 1926). These discrepancies, along with the broad geographic and host range from which Strongyloides specimens were derived in these investigations, lead to differing conclusions and opinions regarding the number of species from primates. 
Formerly, two species names were used for Strongyloides derived from Old World primates - S. fuelleborni Von Linstow, 1905, described from Pan troglodytes and Papio cynocephalus from Africa, and S. simiae Hung and Höppli, 1923 from Asian Macaca sp. The latter species was established on the basis of a smaller oesophagus to total body length ratio in comparison to S. fuelleborni, a lack of prominent constriction behind the vulva of the free-living female, and a 'finely-striated cuticle' (the authors considered S. fuelleborni to have a smooth cuticle) (Hung and Höppli, 1923). However, other authors report that S. fuelleborni and many, if not all other Strongyloides spp., indeed has a striated cuticle though sometimes very difficult to observe (Sandground, 1925; Grove, 1989). As such, the validity of $S$. simiae has been scrutinized by several authors, most of whom regarded the morphological evidence for specific status insufficient or too highly variable for unequivocal species discrimination (Sandground, 1925; Premvati, 1959; Little, 1966a). Staphylococcus simiae became a junior synonym of $S$. fuelleborni, which is the name currently applied to all egg-passing Strongyloides from Old World apes and monkeys (Grove, 1989).

Some biological evidence suggests there may still be valid differences between 'S. simiae' from Macaca spp. and S. fuelleborni. Experimental work by Augustine revealed that crosses between strains derived from Macaca and strains derived from Pan failed to produce offspring - suggesting perhaps that $S$. simiae is indeed a separate species, but nearly impossible to distinguish morphologically from S. fuelleborni (Augustine, 1940). It was also observed that Cebus-derived strains (later designated S. cebus - a species currently considered valid) also failed to cross with the other primate strains, supporting $S$. cebus as a distinct species despite morphologic overlap with S. fuelleborni in several characters (Augustine, 1940; Premvati, 1959). Another potential point of interest is that Von Linstow's original description of $S$. fuelleborni was based on parasites from two African host species (Pan troglodytes and Papio cynocephalus); though no major morphological differences were observed, this could have created inherent variability and lead to assumptions on a lack of host specificity (Von Linstow, 1905). While it has been established that New World and Old World-primate-derived Strongyloides (i.e. S. cebus and S. fuelleborni) are not capable of cross-infection (Faust, 1931), no experiments have been conducted to compare the host specificity within African and Asian S. fuelleborni from different primate hosts.

The distinction between $S$. fuelleborni lineages on the basis of geography is supported by our analysis, where specimens obtained from Africa (Fig. 3, clusters 1 and 2) are clearly distinct from Asian genotypes. The finer division of Asian S. fuelleborni into multiple lineages is also supported (Fig. 3). Strongyloides fuelleborni from Bornean monkeys (proboscis monkeys, long-tailed macaques, silvered leaf monkeys) and orangutans form an outgroup to other genotypes (Fig. 3, clusters 6 and 7), and are also distinct from an undescribed Strongyloides sp. collected from lorises as part of the same Bornean survey (Frias et al., 2018). We also note that the host range of $S$. fuelleborni from Malaysian Borneo (Fig. 3, clusters 6 and 7) and mainland Southeast Asia (Fig. 3, cluster 3) overlap; both genotypes were found in long-tailed macaques, yet the genetic distinction between Bornean and mainland Southeast Asian genotypes is clear. Japanese macaques (Fig. 3, cluster 4) harbour a lineage of $S$. fuelleborni that shares an affinity with isolates from mainland Southeast Asia and Southern Asia (Fig. 3, cluster 3), but these two clusters may still represent distinct groups. We note, however, that the specimens from Japanese macaques were based on an inferred genotype so the analysis was performed a second time, excluding data from Japanese macaques. The structure of the resulting dendrograms remained virtually unchanged following exclusion of this data, indicating that the distinction between $S$. fuelleborni from Malaysian Borneo, Africa and mainland Southeast
Asia/Southern Asia remains supported (Supplementary File S2, Appendix part D). Further sampling of primate Strongyloides across these areas of interest and from other primate species, will help to resolve the true picture of species diversity.

Similar to how the genetic differences observed in host varieties raised important questions on the most appropriate naming of $S$. stercoralis across hosts, the geographic differences we uncovered for S. fuelleborni may also prompt a possible nomenclatural revision. Prior to the availability of molecular analyses, Ashford and Barnish stated that 'In the event of S. fuelleborni is as thus conceived including more than one species, the name S. simiae is available for the parasites of Asian primates' (Ashford and Barnish, 1989). In the 'molecular era', Hasegawa et al. $(2009,2010)$ also remarked on the considerable diversity of $S$. fuelleborni across Asian and African varieties, suggesting that subspecific designations might be indicated for varieties they investigated. Though not possible to definitively confirm the specific status and assign 'S. simiae' or suggest another name for Asian isolates from this work alone, this opens the possibility for such if further characterization continues to support that conclusion. It is also possible that Macaca spp. and other primates may harbour multiple, possibly cryptic, Strongyloides species. Importantly, genetic differences between and among African and Asian primate S. fuelleborni may lead to corresponding differences in zoonotic potential, which could explain the nearly exclusive restriction of human $S$. fuelleborni infections to sub-Saharan Africa. If this is the case, recognizing separate species would be useful if further investigation supports this. Presently, S. fuelleborni genotyping datasets are smaller and less complete compared to those available for S. stercoralis. Additional sequence data from morphologically characterized African and Asian S. fuelleborni would be required from a range of hosts before any taxonomic changes are formalised.

Continuing genetic analyses will aid in resolving a major outstanding question on whether the occurrence of S. stercoralis in animals and humans represents zoonotic spillover (animal to human transmission) or spillback (human to animal transmission). Apart from occasional S. stercoralis infections in great apes living in proximity to infected humans, bona fide S. stercoralis infections, or infections with similar species are very seldom detected in herbivores or the omnivorous primates (Grove, 1989). Therefore, it seems that $S$. stercoralis would have originally evolved in canids or allied taxa (e.g. within the suborder Caniformia) rather than in hominids.

Finally, historic experimentation reveals that two Strongyloides species described from procyonids, S. procyonis and S. nasua, show interesting patterns analogous to observations of $S$. stercoralis of dogs and humans. The raccoon-associated species $S$. procyonis bears close morphological similarities to $S$. stercoralis (see Little, 1966a). In one trial, a short-lived, transient patent S. procyonis infection was successfully established in a human volunteer; one $\operatorname{dog}$ also developed a moderate-duration patent infection (3.5 months) (Little, 1966b). Raccoons may also be susceptible to human $S$. stercoralis - a young raccoon developed a patent infection of moderate duration (92 days) following exposure to filariform larvae collected from an infected human (Johnson, 1962). Strongyloides nasua of coati (Nasua spp.) - is also highly similar to $S$. stercoralis and some authors regard Strongyloides nasua as a synonym for $S$. stercoralis (Moraes et al., 2019). Also, a patent infection was established in a whitenosed coati (Nasua narica) using human-derived S. stercoralis (Sandground, 1926). The observation of morphological overlap and cross-transmissibility among these species of S. stercoralis-like parasites (S. procyonis, S. nasua, S. stercoralis of dogs, and S. stercoralis of humans) supports the idea that the common ancestor of the S. stercoralis-like parasites originated in some ancestral Caniformia carnivore, that adapted to infecting humans sometime 
during the domestication of dogs. This idea was also suggested by Nagayasu et al. (2017) on the basis of S. procyonis being the closest extant relative to $S$. stercoralis, which is also supported by phylogenies constructed from 18S rDNA sequences by Hino et al. (2014). Further genetic characterization of the procyoid parasites (S. procyonis, S. nasua) and other currently undescribed Strongyloides of other caniforms, including both true canids and arctoid mammals (e.g. bears, mustelids), will be necessary to investigate this evolutionary hypothesis.

It follows from the evidence above that $S$. fuelleborni, rather than S. stercoralis, is probably best regarded as the true 'human Strongyloides' that has coevolved with our species, given the primate origin of the former, and that $S$. stercoralis probably represents an evolutionary spillover event. The inability to morphologically distinguish distinct genetic lineages of $S$. stercoralis, the relationship between S. stercoralis and other Strongyloides infecting mammals of the order Caniformia, in conjunction with the clustering of canine $S$. stercoralis (cluster 5) as an outgroup in this analysis, support the hypothesis of Nagayasu et al. (2017) who proposed that the origin of human-infecting $S$. stercoralis was related to the domestication of dogs circa 14,000-6500 years before present (MacHugh et al., 2017). However, our data indicate that some genotypes have seemingly adapted to the human host to a point where they rarely infect dogs (Fig. 2, excluding clusters $4 \& 5$ ). Therefore, these rare cases of dog infection caused by clearly human-adapted genotypes (e.g. Fig. 2, clusters $1-3 \& 6$ ) possibly represent modern and ongoing zoonotic 'spillback'. This highlights that generalizations regarding the role of dogs in human strongyloidiasis are difficult to make without understanding the population structure of this species complex.

\section{Concluding remarks}

Our analysis indicates that the 'two lineage' population structure supposing one zoonotic lineage (genotype A) and a second dogspecific lineage (genotype B) represents an over-simplification of the $S$. stercoralis population structure. Our findings show that a gradient of host permissibility is supported, where one genotype (lineage B; associated with cluster 5, Fig. 2), is dog-specific, producing a natural and distinct outgroup to all of lineage A (Fig. 2, lineage A includes all clusters except 5). Strongyloides stercoralis assigned to clusters 1,2,3 and 6 support a 'spillback' model; otherwise more dog-derived $S$. stercoralis specimens would be expected in these clusters. While available data for $S$. fuelleborni was sparser and less complete in comparison to S. stercoralis, the distinction between African and Asian genotypes seems to support allopatric or vicariant speciation. The separation of Asian genotypes into at least two separate lineages (Fig. 3, clusters 6 and 7 from Malaysian Borneo and clusters 3 and 4 from mainland Asia and Japan), may also be warranted. Close examination of experimental infection and reproductive studies performed by historic investigators generally support the aforementioned trends, as evidenced in our detailed discussion and review of published literature.

The associations between host, geography and genotype reported here, tend to corroborate both early and modern observations that support the proposal that $S$. stercoralis represents a species complex (S. stercoralis sensu lato), and referring to lineage B (Fig. 2, cluster 5) provisionally by a different designation - for example, Strongyloides stercoralis dog genotype, or S. stercoralis canis as proposed by Chandler (1925). Results from further morphological and molecular studies as well as modern in vitro and in vivo biological comparative studies of defined isolates might provide support for S. stercoralis from dogs (specifically, lineage B/ cluster 5) to be renamed as $S$. canis as originally suggested by Brumpt (1922) and later by Augustine (1940).
It appears that Strongyloides fuelleborni is the most appropriate name for species infecting African primates, as this species was originally described from African chimpanzees and baboons (Von Linstow, 1905). However, the genetic distinctions noted here and elsewhere (Fig. 3) (Barratt et al., 2019b) support that future taxonomic revision is needed (Hung and Höppli, 1923) for Strongyloides currently designated as 'S. fuelleborni' infecting Asian primates, although characterization of additional parasite material is needed. It is also possible that African and Asian $S$. fuelleborni groups represent a species complexes of their own, further emphasizing the need for resolving taxonomic relationships among these lineages.

To address the remaining unanswered questions raised here regarding the statuses of $S$. stercoralis and S. fuelleborni as species complexes, we first propose that additional sampling and genotyping is required. This sampling should be focused particularly in areas where there is an ongoing dog and human S. stercoralis sensu lato transmission. Given the strong sampling bias towards S. stercoralis from Southeast Asian countries and Japan, sampling of specimens from other endemic areas would be of great value; data from Africa, the Middle East and South America are particularly sparse. A wider sampling of S. fuelleborni from African humans and other primates is also required. This is especially pertinent to Japanese $S$. fuelleborni for which very few sequences are currently available. In addition to experimental infections to evaluate host specificity, in vitro fertilization/mating experiments such as those performed by Augustine (1940), using genetically characterized isolates could provide critical insight into reproductive isolation and therefore species statuses of Strongyloides populations of interest. Finally, sequencing of additional Strongyloides loci (e.g. as per Nagayasu et al., 2017) could provide increased resolution of genetic relationships when analyses like the one described here are performed.

Supplementary material. The supplementary material for this article can be found at https://doi.org/10.1017/S0031182020000979.

Author contributions. The authors contributed equally to this article. SS and JB prepared the manuscript, reviewed the literature and interpreted the results. JB analysed the data.

Financial support. This research was supported by a grant from the Centers for Disease Control and Prevention Office of Advanced Molecular Detection.

Conflict of interest. The authors have no conflicts of interest to disclose.

Ethical standards. The study uses sequence data that were freely available in a public database (NCBI) and is entirely bioinformatics-based.

Disclaimer. The findings and conclusions in this manuscript are those of the authors and do not necessarily represent the official position of the Centers for Disease Control and Prevention/the Agency for Toxic Substances and Disease Registry.

\section{References}

Ashford RW and Barnish G (1989) Strongyloides fuelleborni. In Grove DI (ed.), Strongyloidiasis: A Major Roundworm Infection of Man. Oxfordshire, UK: Taylor \& Francis, pp. 271-286.

Ashkenazy H, Cohen O, Pupko T and Huchon D (2014) Indel reliability in indel-based phylogenetic inference. Genome Biology and Evolution 6, 31993209.

Augustine DL (1940) Experimental studies on the validity of species in the genus Strongyloides. American Journal of Hygiene. Section D. Helminthology 32, 24-32.

Augustine DL and Davey DG (1939) Observations on a natural infection with Strongyloides In the dog. Journal of Parasitology 25, 117-119.

Barratt JLN, Park S, Nascimento FS, Hofstetter J, Plucinski M, Casillas S, Bradbury RS, Arrowood MJ, Qvarnstrom Y and Talundzic E (2019a) 
Genotyping genetically heterogeneous Cyclospora Cayetanensis infections to complement epidemiological case linkage. Parasitology 146, 1275-1283.

Barratt JLN, Lane M, Talundzic E, Richins T, Robertson G, Formenti F, Pritt B, Verocai G, Nascimento de Souza J, Mato Soares N, Traub R, Buonfrate D and and Bradbury RS (2019b) A global genotyping survey of Strongyloides Stercoralis and Strongyloides Fuelleborni using deep amplicon sequencing. PLoS Neglected Tropical Diseases 13, e0007609.

Basso W, Grandt L-M, Magnenat A-L, Gottstein B and Campos M (2019) Strongyloides Stercoralis infection in imported and local dogs in Switzerland: from clinics to molecular genetics. Parasitology Research 118, 255-266.

Beknazarova M, Barratt JLN, Bradbury RS, Lane M, Whiley $\mathrm{H}$ and Ross $\mathrm{K}$ (2019) Detection of classic and cryptic Strongyloides Genotypes by deep amplicon sequencing: a preliminary survey of dog and human specimens collected from remote Australian communities. PLoS Neglected Tropical Diseases 13, e0007241.

Bisoffi Z, Buonfrate D, Montresor A, Requena-Méndez A, Muñoz J, Krolewiecki AJ, Gotuzzo E, Mena MA, Chiodini PL, Anselmi M, Moreira J and Albonico M (2013) Strongyloides Stercoralis: a plea for action. PLoS Neglected Tropical Diseases 7, e2214.

Brumpt E (1922) Précis de Parasitologie. Paris, France: Masson et Cie.

Castresana J (2000) Selection of conserved blocks from multiple alignments for their use in phylogenetic analysis. Molecular Biology and Evolution $17,540-552$.

Chandler AC (1925) The species of Strongyloides (Nematoda). Parasitology 17, 426-433.

Desportes C (1944) Sur Strongyloides Stercoralis (Bavay 1876) et sur les Strongyloides de primates. Annales de Parasitologie Humaine et Comparée 20, 160-190.

Donath A and Stadler PF (2018) Split-inducing indels in phylogenomic analysis. Algorithms for Molecular Biology 13, 12.

Dorris M, Viney ME and Blaxter ML (2002) Molecular phylogenetic analysis of the genus Strongyloides and related nematodes. International Journal for Parasitology 32, 1507-1517.

Faust EC (1931) Infection experiments in monkeys with human, macaque and Ateles Strains of Strongyloides. Proceedings of the Society for Experimental Biology And Medicine 28, 919-920.

Faust EC (1933) Experimental studies on human and primate species of Strongyloides. II. the development of Strongyloides in the experimental host. American Journal of Hygiene 18, 114-132.

Faust EC and Giraldo LE (1960) Parasitological surveys in Cali, Departamento del Valle, Colombia VI. Strongyloidiasis in Barrio Siloé, Cali, Colombia. Transactions of the Royal Society of Tropical Medicine and Hygiene 54, 556-563.

Freedman DO (1991) Experimental infection of human subjects with Strongyloides species. Reviews of infectious diseases 13, 1221-1226.

Frias L, Stark DJ, Lynn MS, Nathan SK, Goossens B, Okamoto M and MacIntosh AJJ (2018) Lurking in the dark: cryptic Strongyloides in a bornean slow loris. International Journal for Parasitology. Parasites and Wildlife 7, 141-146.

Fuelleborn F (1914) Untersuch. Uber den Infektionsweg bei Strongyloides Und Ankylostomum Und die Biologie dieser Parasiten. Arch. Schiffs-u Tropen-Hyg 18, 26-80.

Fuelleborn F (1927) Über das Verhalten der Larven von Strongyloides Stercoralis, Hakenwürmern und Ascaris lumbricoides im Körper des Wirtes. Arch. Schiffs. $u$. Tropenhyg 31, 1-56.

Galliard H (1939) Recherches sur la strongyloïdose au Tonkin. Rôle des animaux domestiques dans l'étiologie de l'infestation humaine. Annales de Parasitologie Humaine et Comparée 17, 533-541.

Galliard H (1950) Recherches sur l'infestation expérimentale a Strongyloides Stercoralis au Tonkin (1e note). Annales de Parasitologie Humaine et Comparée 25, 441-473.

Galliard H (1951a) Recherches sur l'infestation expérimentale a Strongyloides Stercoralis au Tonkin (2e note). Annales de Parasitologie Humaine et Comparée 26, 67-84.

Galliard H (1951b) Recherches sur l'infestation expérimentale a Strongyloides Stercoralis Au Tonkin (3e note). Annales de Parasitologie Humaine et Comparée 26, 201-227.

Galliard H and Berdonneau R (1953) Strongyloïdose expérimentale chez le chien. Effets de la cortisone. Résultats du test de Thorn à l'hormone corticotrope (ACTH). Annales de Parasitologie Humaine et Comparée 28, 163-171.
Genta RM (1989) Strongyloides Stercoralis: loss of ability to disseminate after repeated passage in laboratory beagles. Transactions of the Royal Society of Tropical Medicine and Hygiene 83, 539-541.

Genta RM and Grove DI (1989). Strongyloides stercoralis infections in animals. In Grove DI (ed.), Strongyloidiasis: A Major Roundworm Infection of Man. London: Taylor \& Francis, pp. 251-270.

Georgi JR and Sprinkle CL (1974) A case of human strongyloidosis apparently contracted from asymptomatic colony dogs. The American journal of tropical medicine and hygiene 23, 899-901.

Golubchik T, Wise MJ, Easteal S and Jermiin LS (2007) Mind the gaps: evidence of bias in estimates of multiple sequence alignments. Molecular Biology and Evolution 24, 2433-2442.

Goodey T (1926) Observations on Strongyloides Fülleborni von Linstow, 1905, with some remarks on the genus Strongyloides. Journal of Helminthology $\mathbf{4}$, $75-86$.

Grove DI (1989) Strongyloidiasis: A Major Roundworm Infection of Man. London: Taylor \& Francis.

Grove DI and Northern C (1982) Infection and immunity in dogs infected with a human strain of Strongyloides stercoralis. Transactions of the Royal Society of Tropical Medicine and Hygiene 76, 833-838.

Hasegawa H, Hayashida S, Ikeda Y and Sato H (2009) Hyper-variable regions in $18 \mathrm{~S}$ rDNA of Strongyloides Spp. as markers for species-specific diagnosis. Parasitology Research 104, 869-874.

Hasegawa H, Sato H, Fujita S, Nguema PPM, Nobusue K, Miyagi K, Kooriyama T, Takenoshita Y, Noda S, Sato A, Morimoto A, Ikeda Y and Nishida T (2010) Molecular identification of the causative agent of human strongyloidiasis acquired in Tanzania: dispersal and diversity of Strongyloides Spp. and their hosts. Parasitology International 59, 407-413.

Hino A, Tanaka T, Takaishi M and Fuji Y (2014) Karyotype and reproduction mode of the rodent parasite Strongyloides venezuelensis. Parasitology 141, 1736-1745.

Hira PR and Patel BG (1980) Human strongyloidiasis due to the primate species Strongyloides fülleborni. Tropical and Geographical Medicine 32, 23-29.

Hung SL and Höppli R (1923) Morphologische und histologische Beiträge zur Strongyloides-Infection der Tiere. Arch. f. Schiffs-u. Tropen-Hyg 27, 118-129.

Jaleta TG, Zhou S, Bemm FM, Schär F, Khieu V, Muth S, Odermatt P, Lok JB and Streit A (2017) Different but overlapping populations of Strongyloides Stercoralis in dogs and humans - dogs as a possible source for zoonotic strongyloidiasis. PLoS Neglected Tropical Diseases 11, e0005752.

Johnson AD (1962) Experimental infection of the raccoon with Strongyloides stercoralis. Journal of Parasitology 48, 332.

Kikuchi T, Hino A, Tanaka T, Aung MPPTHH, Afrin T, Nagayasu E, Tanaka R, Higashiarakawa M, Win KK, Hirata T, Htike WW, Fujita J and Maruyama H (2016) Genome-wide analyses of individual Strongyloides Stercoralis (Nematoda: Rhabditoidea) provide insights into population structure and reproductive life cycles. PLOS Neglected Tropical Diseases 10, e0005253.

Kreis HA (1932) Studies on the genus Strongyloides (Nematodes). American Journal of Hygiene 16, 450-491.

Laymanivong S, Hangvanthong B, Insisiengmay B, Vanisaveth $V$, Laxachack P, Jongthawin J, Sanpool O, Thanchomnang T, Sadaow L, Phosuk I, Rodpai R, Maleewong W and Intapan PM (2016) First molecular identification and report of genetic diversity of Strongyloides Stercoralis, a current major soil-transmitted helminth in humans from Lao People's Democratic Republic. Parasitology Research 115, 2973-2980.

Little MD (1966a) Comparative morphology of six species of Strongyloides (Nematoda) and redefinition of the genus. Journal of Parasitology 52, 6984.

Little MD (1966b) Seven new species of Strongyloides (Nematoda) from Louisiana. Journal of Parasitology 52, 85-97.

Looss A (1911) The anatomy and life history of Ancylostoma Duodenale Dub. A monography. Part II. The development in the free state. Records of the School of Medicine, Egyptian Ministry of Education 4, 163-613.

MacHugh DE, Larson G and Orlando L (2017) Taming the past: ancient DNA and the study of animal domestication. Annual Review of Animal Biosciences 5, 329-351.

Moraes MFD, da Silva MX, Tebaldi JH and Lux Hoppe EG (2019) Parasitological assessment of wild ring-tailed coatis (Nasua nasua) from the Brazilian Atlantic rainforest. International Journal for Parasitology: Parasites and Wildlife 9, 154. 
Nagayasu E, Htwe MPPTH, Hortiwakul T, Hino A, Tanaka T, Higashiarakawa M, Olia A, Taniguchi T, Win SMT and Ohashi I (2017) A possible origin population of pathogenic intestinal nematodes, Strongyloides Stercoralis, Unveiled by molecular phylogeny. Scientific Reports 7, 4844.

Nutman TB (2017) Human infection with Strongyloides Stercoralis and other related Strongyloides species. Parasitology 144, 263-273.

Olsen A, van Lieshout L, Marti H, Polderman T, Polman K, Steinmann P, Stothard R, Thybo S, Verweij JJ and Magnussen P (2009) Strongyloidiasis - the most neglected of the neglected tropical diseases? Transactions of the Royal Society of Tropical Medicine and Hygiene 103, 967-972.

Pampiglione S and Ricciardi ML (1971) The presence of Strongyloides Fülleborni von Linstow, 1905, in man in Central and East Africa. Parassitologia 13, 257-269.

Pampiglione S and Ricciardi ML (1972) Experimental infestation with human strain Strongyloides Fuelleborni in man. Lancet (London, England) 1, 663-665.

Paradies P, Iarussi F, Sasanelli M, Capogna A, Lia RP, Zucca D, Greco B, Cantacessi C and Otranto D (2017) Occurrence of strongyloidiasis in privately owned and sheltered dogs: clinical presentation and treatment outcome. Parasites \& Vectors 10, 345

Premvati G (1959) Studies on Strongyloides of primates: V. Synonymy of the species in monkeys and apes. Canadian Journal of Zoology 37, 75-81.

Sandground JH (1925) Speciation and specificity in the nematode genus Strongyloides. Journal of Parasitology 12, 59-80.

Sandground JH (1926) Biological studies on the life-cycle in the genus Strongyloides Grassi, 1879. American Journal of Epidemiology 6, 337-388.

Sandground JH (1928) Some studies on susceptibility, resistance, and acquired immunity to infection with Strongyloides Stercoralis (Nematoda) in dogs and cats. American Journal of Hygiene 8, 507-538.

Sandosham AA (1952) An investigation into the association of creeping eruption with Strongyloides infection contracted in the Far East. Journal of Helminthology 26, 1-24.

Sato H, Torii H, Une Y and Ooi H-K (2007) A new rhabditoid nematode species in Asian sciurids, distinct from Strongyloides robustus in North American sciurids. Journal of Parasitology 93, 1476-1486.
Schär F, Guo L, Streit A, Khieu V, Muth S, Marti H and Odermatt P (2014) Strongyloides Stercoralis genotypes in humans in Cambodia. Parasitology International 63, 533-536.

Speare R (1989) Identification of species of Strongyloides. In Grove DI (ed.), Strongyloidiasis: A Major Roundworm Infection of Man. London, New York, Philadelphia: Taylor \& Francis, pp. 11-84.

Speare R and Tinsley DJ (1986) Strongyloides felis: an" old" worm rediscovered in Australian cats. Australian Veterinary Practitioner 16, 10-18.

Takano Y, Minakami K, Kodama S, Matsuo T and Satozono I (2009) Cross infection of Strongyloides Between humans and dogs in the Amami Islands, Japan. Tropical Medicine and Health 37, 149-152.

Thamsborg SM, Ketzis J, Horii Y and Matthews JB (2017) Strongyloides Spp. infections of veterinary importance. Parasitology 144, 274-284.

Thanchomnang T, Intapan PM, Sanpool O, Rodpai R, Tourtip S, Yahom S, Kullawat J, Radomyos P, Thammasiri C and Maleewong W (2017) First molecular identification and genetic diversity of Strongyloides Stercoralis and Strongyloides Fuelleborni in human communities having contact with long-tailed macaques in Thailand. Parasitology Research 116, 1917-1923.

Thanchomnang T, Intapan PM, Sanpool O, Rodpai R, Sadaow L, Phosuk I, Somboonpatarakun C, Laymanivong S, Tourtip S and Maleewong W (2019) First molecular identification of Strongyloides Fuelleborni in longtailed macaques in Thailand and Lao People's Democratic Republic reveals considerable genetic diversity. Journal of Helminthology 93, 608-615.

Truszkowski J and Goldman N (2016) Maximum likelihood phylogenetic inference is consistent on multiple sequence alignments, with or without gaps. Systematic Biology 65, 328-333.

Von Linstow OFB (1905) Strongyloides Fülleborni N.sp. Centralblatt für Bakteriologie und Parasitenkunde 38, 532-534.

Wulcan JM, Dennis MM, Ketzis JK, Bevelock TJ and Verocai GG (2019) Strongyloides Spp. in cats: a review of the literature and the first report of zoonotic Strongyloides Stercoralis in colonic epithelial nodular hyperplasia in cats. Parasites \& Vectors 12, 349.

Zhou S, Fu X, Pei P, Kucka M, Liu J, Tang L, Zhan T, He S, Chan YF, Rödelsperger C, Liu D and Streit A (2019) Characterization of a non-sexual population of Strongyloides Stercoralis with hybrid 18S rDNA haplotypes in Guangxi, Southern China. PLoS Neglected Tropical Diseases 13, e0007396. 